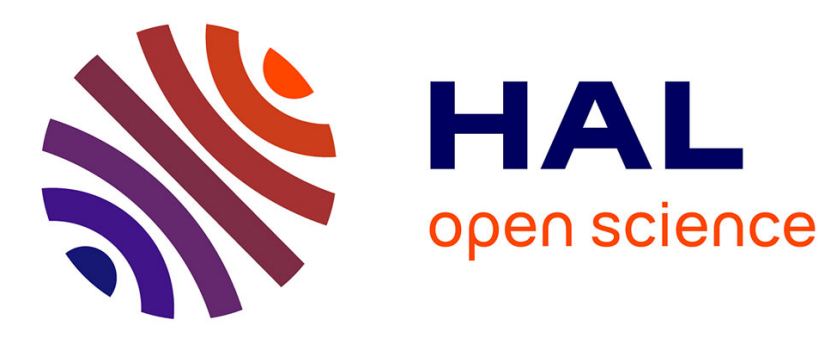

\title{
Mobility Aware and Traffic Adaptive Hybrid MAC Protocol for Collaborative Body Sensor Networks
}

Nadine Boudargham, Jacques Bou Abdo, Jacques Demerjian, Abdallah Makhoul, Christophe Guyeux

\section{To cite this version:}

Nadine Boudargham, Jacques Bou Abdo, Jacques Demerjian, Abdallah Makhoul, Christophe Guyeux. Mobility Aware and Traffic Adaptive Hybrid MAC Protocol for Collaborative Body Sensor Networks. International Journal of Sensor Networks (IJSN), 2020, 32 (3), pp.182 - 196. hal-02993836

\section{HAL Id: hal-02993836 https://hal.science/hal-02993836}

Submitted on 7 Nov 2020

HAL is a multi-disciplinary open access archive for the deposit and dissemination of scientific research documents, whether they are published or not. The documents may come from teaching and research institutions in France or abroad, or from public or private research centers.
L'archive ouverte pluridisciplinaire HAL, est destinée au dépôt et à la diffusion de documents scientifiques de niveau recherche, publiés ou non, émanant des établissements d'enseignement et de recherche français ou étrangers, des laboratoires publics ou privés. 


\title{
Mobility Aware and Traffic Adaptive Hybrid MAC Protocol for Collaborative Body Sensor Networks
}

\section{Nadine Boudargham}

Faculty of Engineering,

Notre Dame University,

Deir El Kamar, Lebanon

E-mail: nboudargham@ndu.edu.lb

\section{Jacques Bou Abdo}

Faculty of Natural and Applied Sciences,

Notre Dame University,

Deir El Kamar, Lebanon

E-mail: jbouabdo@ndu.edu.lb

\section{Jacques Demerjian}

LaRRIS, Faculty of Sciences,

Lebanese University,

Fanar, Lebanon

E-mail: jacques.demerjian@ul.edu.lb

\section{Abdallah Makhoul \& Christophe Guyeux}

FEMTO-ST Institute, UMR 6174 CNRS

Univ. Bourgogne Franche-Comté, Belfort, France

E-mail: \{christophe.guyeux, abdallah.makhoul\}@univ-fcomte.fr

\begin{abstract}
Collaborative Body Sensor Network (CBSN) is formed of several Body Sensor Networks (BSNs) moving in a given area and able to exchange data between each other. One of the challenges in CBSN is to design a Medium Access Protocol that efficiently supports nodes mobility, and at the same time, guarantees immediate delivery of urgent data, and maintains high energy efficiency during regular observation. In this paper, a hybrid Traffic and Mobility Aware MAC (TMA-MAC) is proposed to satisfy CBSN's traffic requirements through adopting a hybrid DS-CDMA/DTDMA technique, and to support CBSN's nodes mobility through ensuring efficient transmission of intra-cluster mobile nodes data and proposing a mechanism to handle inter-cluster nodes joining requests. TMA-MAC was compared to other existing protocols under both traffic and mobility variations. Simulations were conducted using OPNET to evaluate TMA-MAC with respect to packet delay, packet drop percentage, and energy consumption.
\end{abstract}

Keywords: MWSN; CBSN; MAC; DTDMA; DS-CDMA; Mobility; Delay; Packet Drop; Energy Consumption.

\section{Introduction}

The advancements in low power embedded systems, along with the increased need to support mobility in many Wireless Sensor Networks (WSNs) applications such as wildlife monitoring, air and ocean exploration, object tracking, as well as healthcare and military applications, etc. $(1 ; 2 ; 3)$, have led to the emergence of Mobile Wireless Sensor Networks (MWSNs), in which sensor nodes can move freely instead of remaining static as perceived in traditional WSNs. Collaborative Body Sensor Network (CBSN) is one of the MWSN applications that is gaining a lot of research interest lately. CBSN is a network formed of multiple nodes moving in a given area, where every node is a Body Sensor Network (BSN) formed of many wireless sensors that collect physiological data from the human body and send them to the BSN's own Control Unit (CU) or sink node $(26 ; 27)$. These CUs can then inter-communicate between each other to transfer the sensed data to its final destination, i.e. the central Base Station (BS). 
CBSNs allows group instead of individual monitoring, such as supervising the condition of rescue teams, the performance of sports team, and the status of employees in industries, in order to observe group activity, recognize the events identified by many persons, and monitor the health status of multiple persons at the same time (3). An illustration about CBSN general architecture is presented in Figure 1.

In CBSN, there are two types of traffic generated by the composing BSNs: periodic traffic that is produced by the BSNs who are in a state of regular observation, and bursty traffic that is generated by the BSNs in emergency state that happens when the activity of the monitored person or its environment changes suddenly (5). CBSNs present therefore the following challenges:

- Nodes mobility: In CBSN, nodes can move freely in a given area. Some nodes might therefore be mobile, while others remain static. Nodes mobility will lead to dynamic network topology, which adds a challenge to reliable data transmission as mobile nodes might leave their clusters to join new ones.

- Dynamic traffic: During regular observation, BSNs generate low and stable traffic rate (between 120 packets/sec). However, when the $\mathrm{CU}$ of a BSN detects any abnormality during analysis and processing of the received data, it triggers the emergency state, and requests sensor nodes to increase their sampling rate and send more data so it can perform precise recognition. Thus the BSN's traffic rate becomes very high (between 50- 100 packets/sec).

- Strict delay requirements during emergency state: Data transmitted in CBSN can be very critical as it is directly related to people's lives specially for BSNs in emergency state. Therefore during emergency, the main aim is to send critical data within very strict delay requirements (less than $125 \mathrm{~ms})$ and with the lowest packet drops $(6 ; 7)$.

- High energy efficiency requirements during periodic observation: It is very hard to recharge or replace batteries in many CBSN's applications like during fire fighting or in war zones. Thus working on increasing the energy efficiency of the network is very important in CBSN. Therefore, during periodic monitoring, the main aim is to maintain high energy efficiency $(8 ; 9)$

Facing these challenges and guaranteeing reliable transmission of data from the different BSNs to the central BS largely depends on the choice of the Medium Access Control (MAC) protocol. Therefore, it is very important to design a dynamic MAC protocol that is able to adapt to both mobility and traffic variations in CBSN, and is able to address CBSN's traffic demands in maintaining low delay during emergency, and high energy efficiency during periodic monitoring.
Several synchronous and asynchronous MAC protocols were proposed for MWSNs. In synchronous protocols, the clock wakes up nodes at a specific synchronization point of time for a specific period. These protocols aim to reduce collision and re-transmission of data at the expense of clock synchronization requirements between nodes belonging to the same cluster. Whereas asynchronous protocols rely on preamble to declare a transmission. They do not require synchronization between the sensor nodes, as each node works independently without being aware of the active/sleep schedule of the others $(10 ; 2)$. However, the low complexity of these protocols comes at the cost of increased collision, reduced channel availability and idle listening that may occur specially when traffic is high, leading to low delay and energy efficiency $(11 ; 1)$.

This paper considers synchronous MAC protocols, mainly due to their ability to reduce collision rates and re-transmissions, which is essential in CBSNs where traffic can become very high when many nodes enter emergency state instantaneously.

Most synchronous MAC protocols found in literature for MWSNs address the request of mobile nodes to join a new cluster in the next frame period; this is not efficient if the node requesting to join the cluster carries emergency data that should be sent instantly. Also, the suggested protocols do not give mobile nodes priority to send their data before static nodes present in the same cluster; this is not efficient since the transmission link between mobile nodes and the corresponding Cluster Head $(\mathrm{CH})$ can break before the node transmits its data.

On the other hand, most of the synchronous MAC protocols proposed for MWSNs are based on either one or a combination of both Dynamic Time Division Multiple Access (DTDMA) and Carrier Sense Multiple Access with Collision Avoidance (CSMA/CA) protocols $(1 ; 13 ; 14 ; 15 ; 16 ; 17 ; 18 ; 19 ; 20)$. However, as per our previously conducted study in (12), these two protocols do not perform well under heavy traffic conditions, as high delay is induced by large queuing in DTDMA and increased collision rates in CSMA/CA. Therefore, these two protocols will not be able to handle the bursty traffic induced during emergency in CBSN. Whereas this same study proved that Direct Sequence Code Division Multiple Access (DS-CDMA) outperforms both contention-free and contention-based protocols, and induces very low delays when traffic is high.

Therefore in this paper, we present an efficient hybrid DTDMA/DS-CDMA Traffic and Mobility Aware MAC (TMA-MAC) protocol for CBSNs, that aims to address different traffic requirements and at the same time, to support nodes (i.e. BSNs) mobility.

To address different traffic requirements, the proposed scheme uses DS-CDMA to send the emergency traffic generated by both mobile and static nodes, in order to guarantee minimal delay and packet loss during emergency situations, taking advantage of the low delay induced by DS-CDMA in high traffic environment. Whereas it uses DTDMA to handle the 


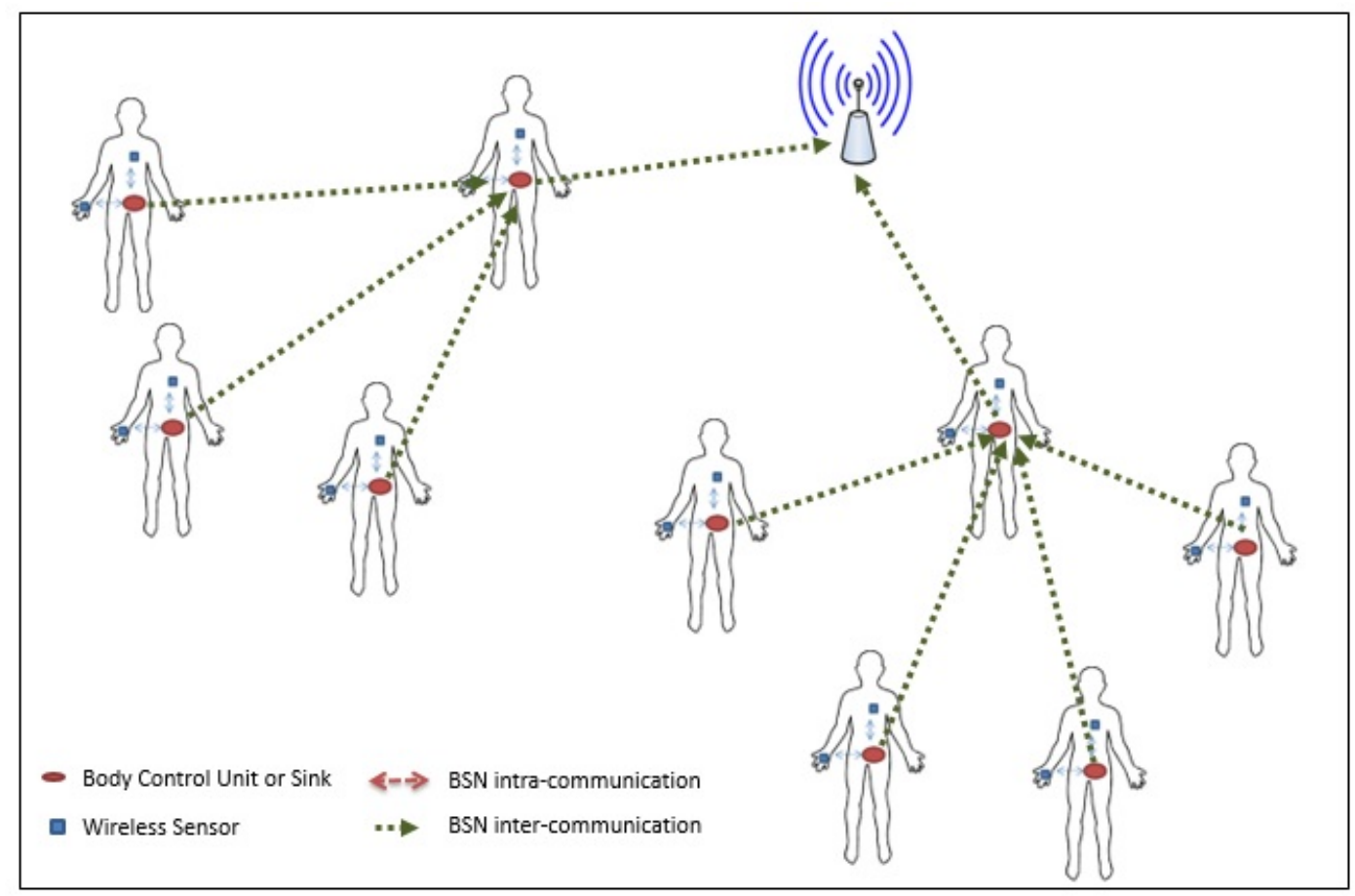

Figure 1: CBSN General Architecture

periodic traffic generated by mobile and static nodes in order to maintain high energy efficiency during regular observations, taking advantage of the low energy consumption characteristic of DTDMA.

The proposed scheme also supports nodes mobility through giving mobile nodes priority over static nodes to send their data as fast as possible before the connection breaks, and through adopting a multichannel mechanism to reduce complexity when mobile nodes need to join new clusters; it also provides a mechanism that allows nodes requesting to join a new cluster immediate channel access when they are holding emergency data to guarantee their instant delivery.

The remaining of the paper is divided as follows: related work is presented in Section 2. An overview of the proposed scheme is provided in Section 3. TMAMAC architecture is described in Section 4. Comparison of the proposed scheme to existing MAC protocols, as well as simulation and discussion of the delay, packet loss and energy performance under various scenarios are provided in Section 5, while conclusion and future work are presented in Section 6.

\section{Related Work}

Few synchronous mobility aware and traffic adaptive MAC protocols are found in the literature. They are mainly based on DTDMA contention-free protocol, of CSMA/CA contention-based protocol, or of a combination of both.

For instance, authors in (13) propose a Mobilityadaptive collision-free MAC (MMAC) that is based on
Traffic-Adaptive Medium Access (TRAMA) protocol. MMAC divides the frame time into two parts: scheduled access and random access. The random access part is used for collecting neighbors information, and the scheduled access part, formed of TDMA slots, is used for data transmission. Unlike TRAMA, MMAC uses a dynamic frame time that is increased or decreased depending on the traffic information, and the expected variation of the number of mobile nodes in two-hop neighborhood. If the number of in-going and out-going nodes is less than a certain threshold, the frame time is increased, otherwise it is decreased to reduce the time waited by the mobile nodes to join the network $(10 ; 11 ; 3)$. Even though MMAC aims to propose an adaptive traffic and mobility approach, it presents shortcomings in both traffic and mobility support. For instance, nodes willing to join a cluster should scan many channels to find the appropriate cluster to join, which increases overhearing and unsuccessful joining attempts to clusters already full. In addition, the protocol does not give priority to mobile nodes in TDMA slots; this is not efficient since mobile nodes should be served quickly as the connection might break. Also, MMAC does not provide a mechanism to handle bursty traffic. For instance, if many nodes carrying bursty traffic want to send their data, then either the frame time should be long enough to hold needed slots, or the nodes will not find available slots due to frame saturation. In both cases, emergency data will not be sent instantly within strict delay requirements. This is in addition to the fact that mobile nodes join requests are not considered in the frame schedule; this is not efficient if the node requesting 
to join the cluster holds emergency data that should be sent instantly.

An adaptive Mobility aware, and Energy efficient MAC (MEMAC) is presented in (1) as a hybrid TDMA/ CSMA protocol. The superframe is formed of two parts: the mini-slots and normal slots. The mini-slots enclose control messages like frame synchronization, random access CSMA period in which nodes compete to send requests for data transmission as well as requests for joining or leaving the cluster, and scheduling period used by $\mathrm{CHs}$ to broadcast scheduling information to corresponding nodes; whereas the normal slots are TDMA based and used to transmit data messages. The frame size in MEMAC is dynamic to ensure the protocol sensitivity to both traffic conditions and mobility. Depending on mobility prediction and location information, MEMAC groups the nodes into Joining nodes "J", Leaving nodes "L", and nodes with data to send "R". Only nodes belonging to "R" are included in the schedule, however the mobility information provided by "J" and "L" is used to adapt the frame time. If the number of nodes in "R" is greater than those in "J" and "L" combined together, the frame size is increased, otherwise, the frame length is decreased. MEMAC have the same shortcomings as MMAC, with the addition to the fact that nodes have to compete to reserve time slots which might increase transmission delays due to high contention that occurs in heavy traffic.

In (14), authors propose a Mobility adaptive Hybrid MAC (MH-MAC) protocol. MH-MAC divides the frame into static and mobile slots. Static slots are scheduledbased, whereas mobile slots are contention-based to avoid TDMA scheduling overhead. Based on mobility estimation algorithm, nodes can determine their mobility level. If the number of mobile nodes is greater than a certain threshold, the network is considered mobile, the frame time is reduced, and the number of mobile slots is increased with respect to static slots; whereas if the number of mobile nodes is less than the threshold, the network is considered static, the frame time is increased, and the number of static slots is increased with respect to mobile slots. The drawback of MH-MAC is that static slots are assigned before mobile slots which is inefficient as mobile nodes should be served fast before losing transmission link. Also, the protocol is unable to handle emergency or bursty traffic as it adopts TDMA for static nodes and CSMA for mobile nodes, and both of them are not efficient in heavy traffic. Thus static nodes might experience large packet transmission delays due to TDMA queuing, and mobile nodes may not succeed to send their data due to the high contention that occurs in heavy traffic.

Authors in (15) present MobiSense : a power efficient, micro-mobility MAC protocol. Mobisense is based on TDMA. Its superframe is formed of synchronization slots, fixed length downlink slots used by $\mathrm{CHs}$ to broadcast information to nodes, variable length uplink slots used by nodes to transmit their data to the $\mathrm{CH}$, and access mini-slots or Access Window (AW) used by mobile nodes to send their join requests to $\mathrm{CHs}$ without contention. The length of the downlink slots period depends on the nodes' traffic demands, and the AW is reduced as the cluster size grows. In Mobisense, $\mathrm{CHs}$ advertise over common control channel information about their cluster size and the corresponding data transmission channel; mobile nodes who want to join a new cluster listen to this common channel, which will speed up their joining process and prevents unsuccessful join attempts. Even though Mobisense presents many solutions for efficient mobility support, it is unable to handle bursty traffic efficiently since it uses TDMA in data transmission, and will not be therefore able to transmit emergency data generated by multiple nodes within strict delay requirements. Also, nodes willing to join a cluster will have to wait for the AW to send joining requests over the access mini-slots, which is not efficient if these nodes are holding urgent data that should be granted immediate channel access to transmit their packets.

A Time Sharing Energy Efficient Congestion control (TSEEC) MAC is proposed in (16). TSEEC is a TDMA based MAC protocol. It uses Load Based Allocation (LBA) technique to assign dynamic time slots to different nodes; LBA uses statistical information about sensor nodes like node memory, battery, and location information to compute a corresponding weighted value. The weighted value depends on the node's Time of Arrival (ToA), battery lifetime, location, and priority value. Time slots are then assigned based on the obtained weighted value of every node. TSEEC also uses Time Allocation Leister (TAL) technique to handle time slots that are either assigned to nodes that are no longer carrying data, or to leaving nodes: it assigns the free slots to mobile nodes joining the cluster, or it broadcasts the free slot message to neighboring mobile nodes to shift back their own time slots. Both LBA and TAL techniques decrease the network congestion and increase energy conservation; however, the protocol uses TDMA slot allocation which leads to long waiting time when the traffic becomes heavy in the presence of many nodes holding emergency data. Also, mobile nodes looking for new cluster will have to scan many channels to find the appropriate cluster, which induces higher delay and more complexity.

Other proposed schemes like MS-MAC (17), EMSMAC (18), AM-MAC (19), and MD-SMAC (20) are synchronous protocols with common active/sleep period. These schemes are based on CSMA/CA; they compute a random value to determine the access period of every node, and nodes that are active at the same time follow CSMA/CA scheme to gain channel access. These protocols are simple in general; however, they are not efficient when the traffic becomes heavy as the contention between active nodes increases and leads to high collision rates, and thus high delays and energy consumption.

The above discussion shows that the currently proposed protocols are not suitable for CBSNs where both traffic variations and mobility should be handled 
efficiently. The main shortcomings of these schemes are their inability to guarantee prompt delivery of emergency data due to DTDMA and CSMA/CA low efficiency in heavy traffic, and their failure in providing an efficient mechanism to handle urgent join requests to guarantee fast delivery of mobile data.

For this reason, we propose a hybrid Traffic and Mobility Aware MAC protocol (TMA-MAC). The proposed scheme addresses CBSN's traffic requirements in ensuring reliable delivery of emergency (bursty) traffic generated by static or mobile nodes within strict delay requirements, while increasing the network energy efficiency during transmission of periodic traffic. The proposed scheme also provides an efficient mobility support mechanism that addresses joining requests and data transmission of mobile nodes holding both emergency and periodic traffic.

\section{Hybrid TMA-MAC Protocol Overview}

We consider a CBSN formed of $m$ nodes, i.e. BSNs, grouped into different clusters and distributed in $n x$ $n$ area. Some of these nodes are mobile while others are static. During regular observations (non-emergency cases), nodes generate periodic traffic with constant packet rate ranging between 1 and 20 packets/sec. When some nodes enter into emergency state due to sudden variation in their activity or environment, they will generate a burst of traffic and their corresponding packet rate will significantly increase to reach 100 packets/sec.

The proposed TMA-MAC addresses both traffic and mobility requirements of CBSNs.

\subsection{Traffic Awareness}

Dynamic Time Division Multiple Access (DTDMA) is a scheduled contention-free protocol, in which dynamic time slots are allocated to nodes based on the corresponding traffic rate. DTDMA generates low delay in periodic traffic environments, however it is unable to handle very high traffic as it induces significant delay due to queuing. The main strength of DTDMA is its low energy consumption in periodic traffic, since nodes do not face collisions, idle listening and overhearing issues, as they send their data in allocated time slots and remain inactive all the other times $(21 ; 22)$. Direct Sequence Code Division Multiple Access (DSCDMA) is a contention-free protocol is which different nodes are assigned different spreading codes to allow concurrent transmission of their data over the same channel. The assigned spreading code is multiplied by the node's original signal to generate the encoded data. The computational requirements to encode the packets increases DS-CDMA energy consumption; however, the main strength of this protocol is its high delay efficiency even in bursty traffic environment (12). Thus to handle various traffic requirements, TMA-MAC adopts a hybrid DTDMA/DS-CDMA approach to combine the advantages of both DTDMA and DS-CDMA Protocols.

During regular observation, the traffic generated by nodes in a cluster is low and periodic. therefore, nodes send their data packets to the corresponding $\mathrm{CH}$ in allocated time slots using DTDMA mechanism to take advantage of the high delay and energy efficiency of DTDMA in periodic traffic. When some nodes in the cluster enter into emergency state, their traffic rate significantly increases, and at the same time, their data is considered critical and should be delivered to the $\mathrm{CH}$ within strict delay requirements. For this reason, TMAMAC dynamically allocates slots for emergency traffic, in which data from various nodes can be transmitted simultaneously by assigning them different spreading codes using DS-CDMA. This will guarantee concurrent delivery of urgent data with the lowest delay over the same time slots, due to high delay efficiency of DSCDMA in heavy traffic environment (12). The remaining nodes carrying periodic traffic will keep on transmitting their data one after another in their allocated slots using DTDMA mechanism. Therefore, only nodes carrying emergency traffic will be assigned different codes, which will limit the energy consumption in the network.

Therefore, TMA-MAC succeeds in addressing CBSN traffic requirements through the following features:

- It adapts to CBSN traffic variations through dynamically allocating time slots to nodes based on traffic rates.

- It is able to address CBSN's emergency traffic requirements in delivering urgent data reliably with minimal delays.

- It is able to address CBSN's periodic traffic requirements in inducing low delay and energy consumption.

\subsection{Mobility Support}

The proposed TMA-MAC considers two types of mobility (28): intra-cluster mobility, in which mobile nodes remain within their cluster, and inter-cluster mobility in which mobile nodes leave their cluster and look for other clusters to join. TMA-MAC supports nodes mobility by ensuring efficient and reliable transmission of both periodic and emergency data held by intra-cluster and inter-cluster mobile nodes, through the following procedures:

1. Intra-cluster mobile nodes carrying periodic traffic send their data in allocated time slots using DTDMA; however, they are given priority over the equivalent static nodes to ensure fast data delivery before the transmission link is lost; mobile nodes with the lowest Received Signal Strength Indicator (RSSI) are assigned slots first.

2. Intra-cluster mobile nodes carrying emergency traffic are assigned different spreading codes to 
enable them to transmit their data simultaneously over the emergency slots, along with the equivalent static nodes.

3. In order to ensure simple and efficient joining mechanism for inter-cluster mobile nodes to new clusters, TMA-MAC adopts a common control channel as in (15) over which $\mathrm{CHs}$ send corresponding cluster information, to allow intercluster nodes to quickly and successfully choose their new cluster.

4. When an inter-cluster mobile node carries emergency traffic, it listens to the common control channel to choose the most appropriate cluster to join. To join the new cluster, the node sends join request in the Emergency Join Request (EJR) mini-slots distributed between the DTDMA slots throughout the frame time, to address their request quickly. Once the join request is accepted, the frame time is interrupted to allow prompt delivery of this urgent traffic. The $\mathrm{CH}$ distributes the new schedule to the cluster nodes, in which urgent data is sent first, followed by periodic traffic of mobile nodes, and the periodic traffic of static nodes.

5. Inter-cluster mobile nodes carrying periodic (nonemergency) traffic can send their joining requests over a specific Contention Assess Period (CAP) placed at the end of the frame time. Data generated by these nodes is addressed in the next frame time schedule.

\section{TMA-MAC Architecture}

\subsection{Superframe Structure}

The superframe of the data communication channel of TMA-MAC is presented in Figure 2.

It is formed of a Beacon Field (BF), Emergency Slots (ES), Mobile nodes Slots (MS), Static nodes Slots (SS), a Contention Access Period (CAP), and several Emergency Join Request (EJR) mini-slots inserted

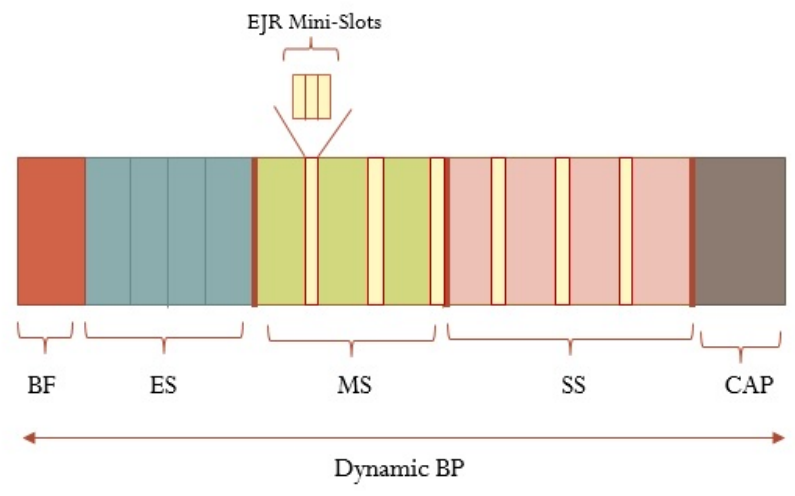

Figure 2: TMA-MAC Superframe Structure between some Mobile and Static Slots (MS and SS). The Beacon Period (BP) represents the superframe length or duration.

The $\mathrm{BF}$ is used by the $\mathrm{CH}$ to convey synchronization information to the corresponding cluster nodes, along with other information like slot allocation in ES, MS, SS periods, and spreading codes. To synchronise nodes, TMA-MAC uses Reference Broadcast Synchronization (RBS) method (29), in which nodes use the packets time of arrival as a reference for clock synchronization when receiving the synchronization packets. This synchronization mechanism is adopted since it is simple and used in many MWSN protocols $(23 ; 15)$.

ES is used by both static and intra-cluster mobile nodes carrying emergency (bursty) traffic. These slots allow transmission of simultaneous packets through assigning them different spreading codes using DSCDMA to ensure fast delivery of the urgent data. The length of the ES period is dynamic and depends on the highest packet rate generated by the emergency nodes. When all nodes are in periodic observation states, the superframe will not enclose an ES period, however, when one or more nodes enter into emergency state, the ES period increases to serve the highest generated data rate. However, the ES period is limited to $120 \mathrm{~ms}$, to guarantee delivery of urgent data within their delay requirements (125 ms) (9).

MS are used by intra-cluster mobile nodes holding periodic traffic to send their data one after another using DTDMA mechanism. These Mobile Slots (MS) are given priority over Static Slots (SS) to ensure fast their data transmission before the link breaks. The MS period is also dynamic and depends on the packet rates of every node (i.e. the number of slots needed for every node).

SS are used by intra-cluster static nodes holding periodic traffic. These slots come after the MS since the probability for link breakage is lower. As in MS, static nodes send their periodic data in SS using DTDMA, and has a dynamic period depending on the needed number of slots for every node.

CAP is used inter-cluster mobile nodes carrying periodic traffic to send join request to the cluster. If the join request is accepted by the $\mathrm{CH}$, the corresponding data will be transmitted in the MS of the next frame. The CAP is also used by the intra-cluster mobile nodes to announce their departure from the cluster.

EJR mini-slots are used by inter-cluster mobile nodes holding urgent traffic to send join request to the cluster. These mini-slots are distributed between time slots of MS and SS periods to allow nodes carrying critical data to join the cluster the soonest. The structure of EJR frame, presented in Figure 3, is formed of two parts: Data and ACK fields. The data part is used by the intercluster mobile node to send its join request, whereas the $\mathrm{ACK}$ part is used by the $\mathrm{CH}$ to send the join request decision.

The BP in TMA-MAC is dynamic and depends on the ES, MS, SS periods as well as on the number of EJR mini-slots. However, it is bounded by a maximum size is 


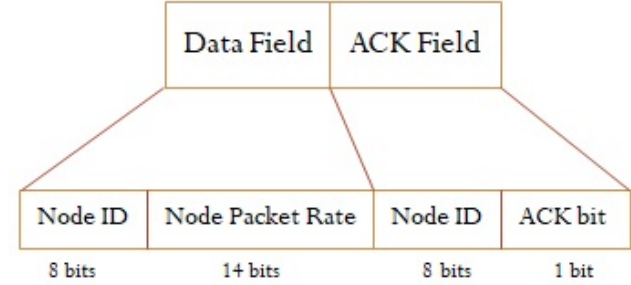

Figure 3: EJR Mini-Slot Frame Structure

set to $240 \mathrm{~ms}$ in order to satisfy the delay requirements of regular traffic for BSNs (9).

\subsection{Slot Allocation Procedure}

The optimal number of slots that should be assigned for every node in a cluster is given by:

$$
\text { Slots }=\frac{R \cdot B P}{P P S}
$$

where:

$$
\begin{aligned}
& R: \text { Packet Rate } \\
& B P: \text { Beacon Period } \\
& P P S: \text { Number of Packets Per Slot }
\end{aligned}
$$

and

$$
P P S=\frac{\text { Slot Duration }}{\text { Duration of Packet }}
$$

Equation (1) shows that the number of allocated slots is directly proportional to the traffic (packet rate). The slot allocation mechanism provided by this equation is efficient, as assigning more slots will not improve the performance, since it helps nodes attaining the highest throughput $(5 ; 24)$. The dynamic slot allocation algorithm for static and mobile nodes in emergency state, is presented in Algorithm 1. If only one node is in emergency state, thus carrying critical data, the $\mathrm{CH}$ computes the corresponding number of slots needed using equation 1 based on the packet rate generated by the node. However, if more than one node is in emergency state, the $\mathrm{CH}$ assigns a spreading code to each of these nodes, and uses equation 1 to compute the required number of slots based on the highest received packet rate. In both cases, the number of allocated slots is bounded by a maximum number $s_{\max }$ to guarantee the delivery of urgent data within their strict delay requirements (120 ms). The $\mathrm{CH}$ then allocates the computed number of slots in the ES period, and updates the information about spreading codes and slot allocation in the BF (5).

The dynamic allocation procedure for mobile nodes in periodic observation state, thus carrying regular data, is shown in Algorithm 2. As described in Algorithm 1,

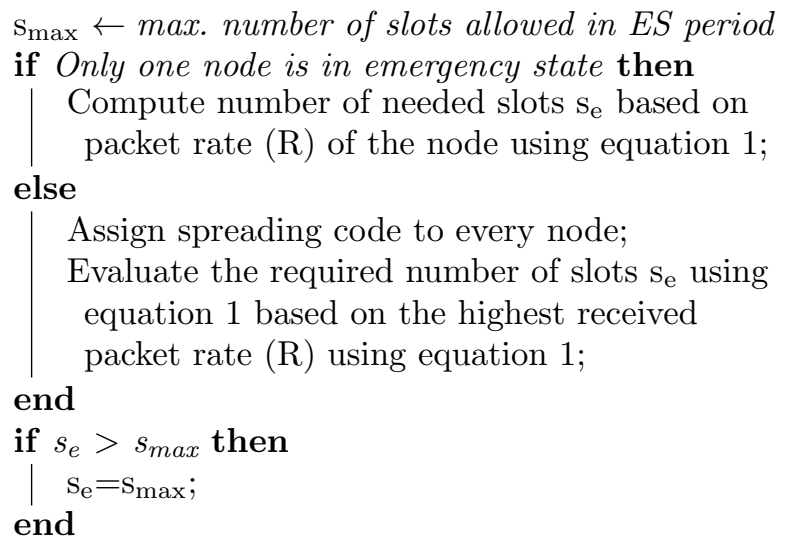

Allocate $\mathrm{s}_{\mathrm{e}}$ for in ES period;

Update the information about spreading codes and slot allocation in the BF;

Algorithm 1: Dynamic Slot Allocation for Nodes in Emergency State

when a node is in emergency state, it will be assigned a spreading code to enable simultaneous delivery of its data over the ES slots. However, when this node gets back to regular/ periodic observation, the $\mathrm{CH}$ frees it from the previously assigned spreading code. The $\mathrm{CH}$ then computes the number of slots needed for every mobile node based on their corresponding packet rates using equation 1, to allocate them in the MS period. Mobile nodes keep track of the RSSI level of the packets received from the $\mathrm{CH}$; Consequently, nodes with the lowest RSSI level are given priority over others and are allocated slots first in the MS period to ensure their fast data transmission before the link breaks. The $\mathrm{CH}$ then updates the information about spreading codes and slot allocation in the BF.
for every mobile node $m_{i}$ carrying periodic data do if $m_{i}$ is previously assigned a spreading code then | Release spreading code from $\mathrm{m}_{i}$; end Compute number of needed slots $\left(\mathrm{s}_{\mathrm{mi}}\right)$ based on the packet rate $(\mathrm{R})$ of $\mathrm{m}_{\mathrm{i}}$ using equation 1 ; Allocate $\mathrm{s}_{\mathrm{mi}}$ in MS period;; if $R S S I_{m j}<R S S I_{m i}$ then I Allocate $s_{\mathrm{mj}}$ before $\mathrm{s}_{\mathrm{mi}}$; end

end

Update the information about spreading codes and slot allocation in the BF;

Algorithm 2: Dynamic Slot Allocation for Mobile Nodes in Regular State

The dynamic slot allocation process for static nodes carrying periodic traffic is presented in Algorithm 3. Similar to mobile nodes carrying periodic traffic, the $\mathrm{CH}$ releases any previously assigned spreading code, and computes the required number of slots using equation 
1. The $\mathrm{CH}$ then allocates the corresponding slots in the SS period, and updates the slot allocation and spreading code information in the BF.

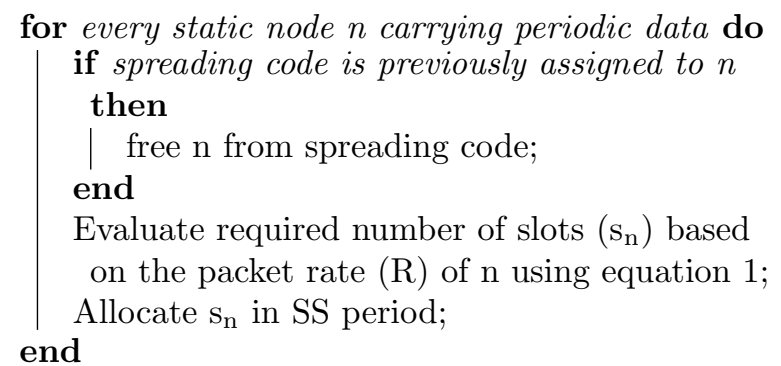

Update the information about spreading codes and slot allocation in the BF;

Algorithm 3: Dynamic Slot Allocation for static Nodes in Regular State

\subsection{Identification of Intra-cluster Mobile Nodes}

Mobile nodes within a cluster are identified based on the RSSI level of the synchronization packet received from the $\mathrm{CH}$. The nodes keep track of the RSSI variations. Based on Nordic nRF24L01 radio transceiver that is widely used in BSNs, the RSSI level that guarantees reliable transmission is $-64 \mathrm{dBm}(25)$. Below this value, transmission links become unreliable. Thus in the proposed scheme, if the RSSI level is found to be decreasing over time to reach a threshold of $-55 \mathrm{dBm}$, the node inside the cluster is considered to be mobile and should be served quickly before the value decreases further and the link breaks.

\subsection{Computation of Number of EJR Mini-Slots}

As stated earlier, EJR mini-slots are used by inter-cluster mobile nodes carrying critical trafc to send cluster join requests. Multiple mini-slots are distributed between the time slots of MS and SS period, to allow these nodes to send join requests as soon as they enter the cluster area, and transmit their critical data promptly without further delays. Nodes willing to join a cluster choose EJR mini-slots randomly to send their join request as shown in Figure 4. Random selection of minislots significantly reduces the probability of collision in moderate contention situations when multiple nodes want to join a cluster simultaneously.

Increasing the number of mini-slots would increase the opportunity for join requests, and speed out the joining operation and data transmission of urgent data; But at the same time, too many mini-slots would increase the $\mathrm{BP}$ or frame time, which would delay the data transmission of the other nodes, specially that these mini-slots might not be used when inter-cluster mobile nodes do not hold emergency data. For this reason, the number of EJR mini-slots is dynamically computed in
TMA-MAC in a way not to exceed $5 \%$ of the frame time to maintain a reasonable number of mini-slots without considerably affecting the frame time. For instance, Figure 3 shows that approximately 4 bytes are needed for every EJR mini-slot. Considering a channel rate of $250 \mathrm{kbits} / \mathrm{s}$, the EJR mini-slot duration will be $0.128 \mathrm{~ms}$. The BP in TMA-MAC is dynamic as it depends on the ES, MS, SS periods. Considering a BP of $128 \mathrm{~ms}$, the number of EJR mini-slots should not lead to more than $5 \%$ increase in the BP, that is $6.4 \mathrm{~ms}$. Therefore for this $\mathrm{BP}$, the superframe can include a maximum of 50 minislots distributed between the MS and SS slots. The BP is exempted from mini-slots when it becomes saturated and can no longer accept join requests.

\subsection{Inter-cluster Communication Operation}

For efficient inter-cluster communication, TMA-MAC uses a common control channel, and different data transmission channels (one data transmission channel per cluster).

The common control channel is used by the $\mathrm{CHs}$ to send information related to their clusters, like number of cluster nodes, number of available mini-slots, BP, and channel used for data transmission. This common channel will help inter-cluster mobile nodes to join a new cluster quickly, as they only need to listen to one channel instead of scanning many channels. This would also decrease overhearing and reduce the probability of failed join attempts.

In TMA-MAC, the handover decision is taken by the mobile node when the RSSI level of the packets received from the current $\mathrm{CH}$ becomes less than $-64 \mathrm{dBm}$; In this case, the link with the current cluster is considered to be no longer reliable, thus the node announces its departure in the CAP of its current cluster's superframe, and searches to join a new cluster with a reliable link.

The Communication operation for join requests is illustrated in Figure 5.

When a mobile node with critical traffic decides to leave its cluster, it listens to the common channel and selects the cluster with the lowest number of nodes, and available EJR mini-slots. It then sends its address, and

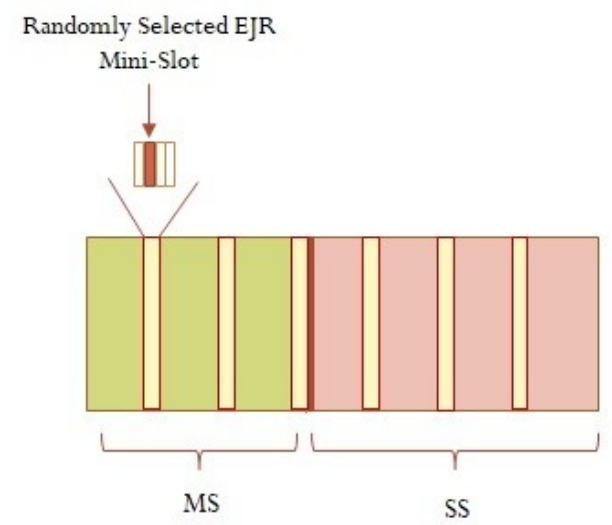

Figure 4: EJR Mini-Slot Random Selection 


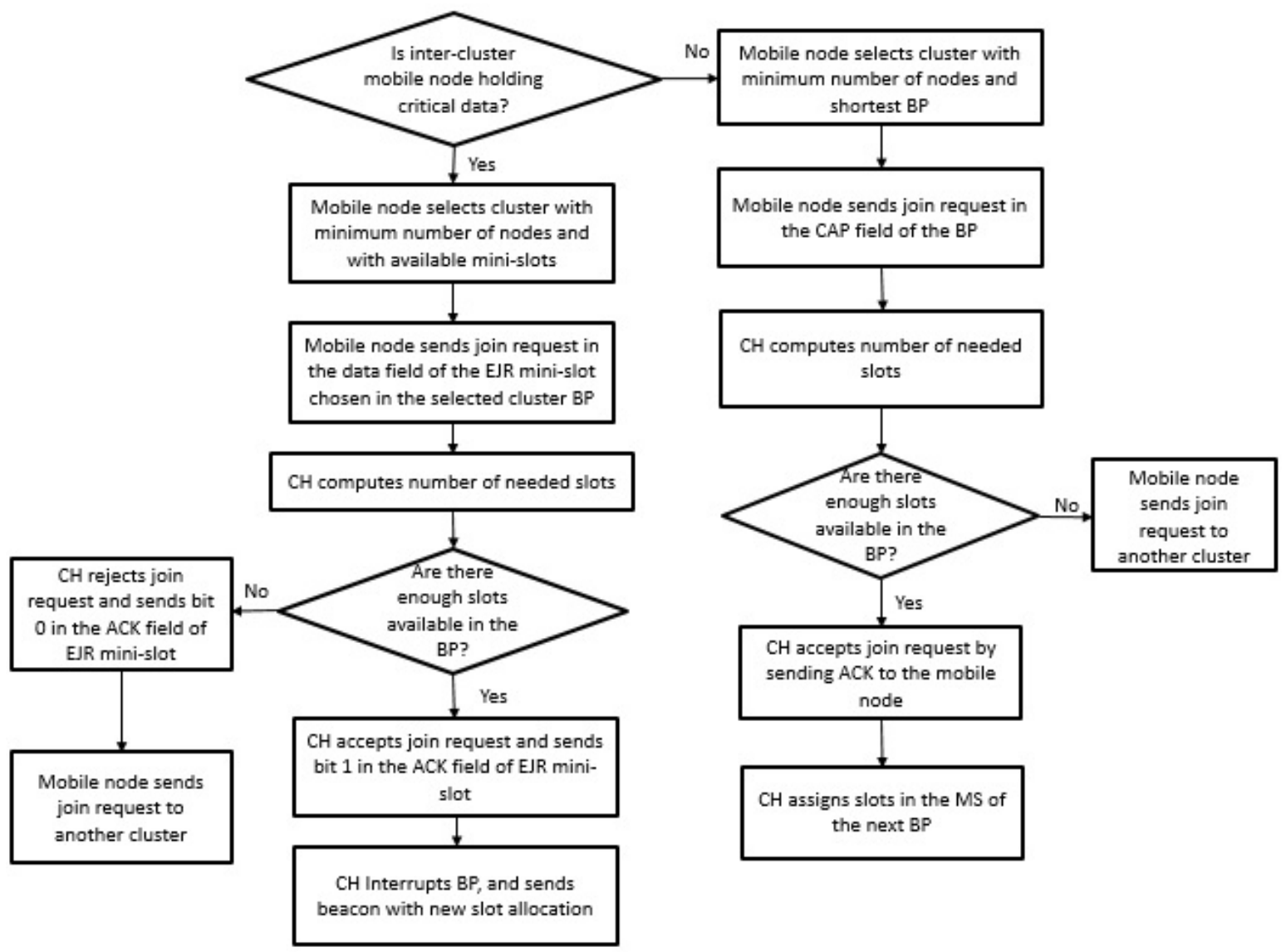

Figure 5: Block Diagram of TMA-MAC Inter-cluster Join Request Operation

corresponding packet rate in the data part of the EJR mini-slot that it selects randomly among the first set of mini-slots it finds in the BP of the chosen cluster. The $\mathrm{CH}$ evaluates the number of slots needed to serve the node based on the received packet rate using equation 1. If it can serve the node (i.e. it has enough slots), it sends a positive acknowledgement (bit 1) in the ACK field of the EJR mini-slots. The $\mathrm{CH}$ then interrupts the BP, and sends a beacon packet that includes synchronization information and new slot allocation schedule, in which it grants this node immediate channel access to send its critical data promptly at the beginning of the new Beacon Period. The remaining nodes will proceed in transmitting their data in the newly allocated time slots. If several nodes send join requests over consecutive EJR mini-slots, they will transmit their data simultaneously over the same slots by assigning them different spreading codes. Even though sending a new beacon constitute an additional overhead, this mechanism is performed when inter-cluster nodes hold critical data only, to ensure their transmission within strict delay requirements. In case the $\mathrm{CH}$ finds that it can not serve the node due to superframe saturation, it rejects the join request through sending a negative acknowledgement (bit 0) in the ACK field of the EJR mini-slot, so the node can send its join request to another cluster.
As for the mobile node holding regular traffic and looking for a new cluster to join, it listens to the common channel and selects the cluster with the lowest number of node and shortest BP. It then sends its join request in the CAP. The $\mathrm{CH}$ checks for slots availability through computing the required number of slots using equation 1. If slots are available, it sends an ACK to the node indicating join request acceptance, and assigns these slots in the MS period of the next BP.

\subsection{Operational Example}

To sketch the operation of the proposed TMA-MAC, consider a CBSN formed of many patients (BSNs or nodes) in a hospital, to monitor their health condition. These nodes are grouped into several clusters. Let us assume that one cluster is formed of a $\mathrm{CH}$ and eight nodes, where the $\mathrm{CH}$ is static, and the cluster nodes can be static or mobile. In this example, we assume that four of these nodes are static and the other four are mobile. Figure 6 a shows the slot allocation procedure when all nodes are in regular observation state and thus carrying periodic traffic. Mobile nodes are given priority over static nodes, and are allocated slots first in the MS period. Also, mobile nodes with lower RSSI levels are given priority over the ones with higher RSSI values to guarantee their delivery before the connection breaks. Static nodes are allocated slots next in the SS period. 
In this case, ES period is non-existent. The number of slots reserved for every node is computed using equation 1. For instance, for a payload size of 32 bytes and channel rate of $250 \mathrm{kbps}$, the packet will need $1.024 \mathrm{~ms}$ to be transmitted via every node's radio. Using Nordic nRF24L01 radio transceiver, the time needed to activate its transmission mode is $0.16 \mathrm{~ms}$ (25); therefore the total packet duration is $1.184 \mathrm{~ms}$. Therefore, if the slot duration is $5 \mathrm{~ms}$, then 4 packets can be accommodated in every slot. For a BP of $240 \mathrm{~ms}$, the number of slots needed to send the periodic traffic is either 1 or 2 depending on the corresponding packet rate $(2$ slots for a rate of 20 packets/sec, and 1 slot for a rate of 5 packets/sec for example).

If one or more patients experience sudden change in their activities, like fainting, or facing sudden drop of heart rate, they enter into emergency state; their data becomes bursty and at the same time very critical and should be delivered very quickly to take appropriate actions. Assuming in this example that one mobile person (node 4) and another static person (node 5) enter into emergency state simultaneously; In this case, data of these two persons will be encoded and transmitted concurrently over the same time slots allocated in the ES period of the $\mathrm{BP}$ as shown in Figure 6b. The number of allocated slots depends on the highest packet rate generated by these nodes. For instance, if node 2 generates a traffic of 60 packets / sec and node 4 generates a traffic of 100 packet/sec, the number of slots will be computed based on node's 4 traffic rate, that is 6 slots. The duration of the ES period will be therefore $30 \mathrm{~ms}$, which is less than the maximum allowed period (120 $\mathrm{ms}$ ) to guarantee transmission of the critical data within their delay requirement. The remaining nodes keeps on transmitting their data in their allocated slots in the MS and SS periods respectively.

Now assuming that a mobile patient (node 9) enters in emergency state, but lost the reliable connection with his assigned cluster, and decides to join this cluster after listening to the common channel. Since node 9 is carrying critical data, it sends join request over the first EJR mini-slot. Assuming that the corresponding packet rate is 100 packets/sec, then the $\mathrm{CH}$ evaluates the number of needed time slots to be 6 slots. Since the BP is not saturated (still did not reach $240 \mathrm{~ms}$ ), the join request will be accepted by the $\mathrm{CH}$; the $\mathrm{BP}$ will be interrupted to allow prompt delivery of node 9 data as shown in Figure 6c. All the other mobile and static nodes with periodic data will continue sending their data after node 9 data transmission is over.

\section{Evaluation of TMA-MAC}

To evaluate the performance of the proposed TMAMAC protocol, the suggested scheme is compared to ME-MAC (1), Mobisense (15), and TSEEC (16) MAC protocols. ME-MAC is chosen since it is a synchronous traffic adaptive protocol, that employs variable frame

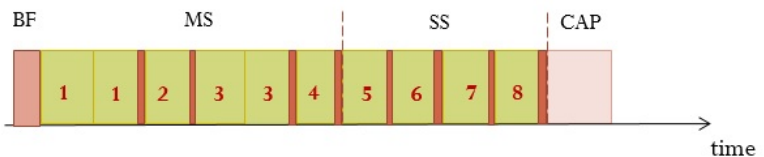

(a) Periodic Traffic Case

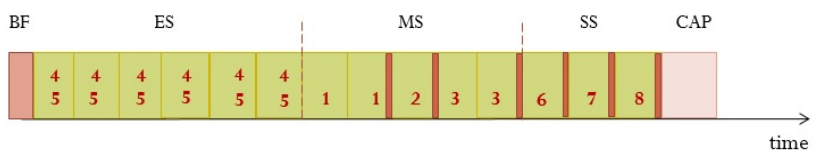

(b) Case of Two Nodes in Emergency State

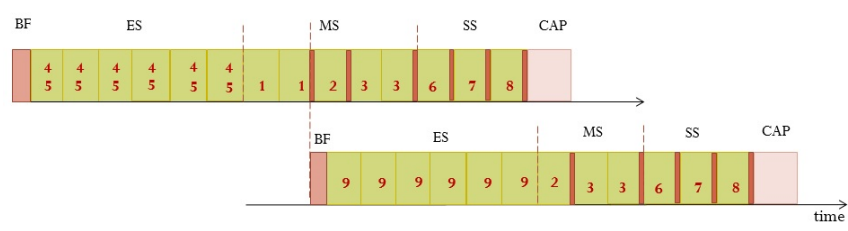

(c) Case of Mobile Node with Emergency Traffic Joining the Cluster

Figure 6: Slot Allocation Operational Example

size based on mobility and traffic demand, which is close to the concept of the proposed scheme; Mobisense is chosen since it is a synchronous traffic adaptive protocol that adopts a common control channel and multiple data communication channels technique which is used in the proposed TMA-MAC; and TSEEC protocol is used since it is a recent traffic adaptive protocol that proposes a congestion control mechanism through two concepts: assigning slots to the nodes based on a computed weighted value (i.e. different priorities are given to different nodes), and allowing slots sharing; these concepts are also close to the proposed mechanism. The average delay, the packet drop percentage and the energy consumption of the four schemes are simulated, and the results are discussed to assess the performance of the TMA-MAC with respect to other existing traffic and mobility aware techniques.

\subsection{Simulation Parameters}

In the simulation, we consider 50 nodes (BSNs) randomly distributed in a $500 \mathrm{~m}^{2}$ area. These nodes can be either static or mobile, and are grouped in different clusters in which $\mathrm{CHs}$ are fixed, whereas cluster nodes are a mix of static and mobile nodes. To form clusters, a LEACH variant protocol is used in which $\mathrm{CHs}$ are only chosen among the static nodes only, based on the 


\begin{tabular}{||l|l||}
\hline Parameter & Value \\
\hline \hline Number of Nodes & 50 \\
\hline Simulation Area & $500 \mathrm{~m}^{2}$ \\
\hline CHs state & Static \\
\hline Nodes state & Static or Mobile \\
\hline Mobility Model & Random Way Point \\
\hline Packet Rate for Regular Traffic & $1-20$ packets/sec \\
\hline Packet Rate for Emergency Traffic & 100 packets $/ \mathrm{sec}$ \\
\hline Payload Size & 32 Bytes \\
\hline Slot Duration & 5 ms \\
\hline Channel Rate & $250 \mathrm{Kbps}$ \\
\hline
\end{tabular}

Table 1 Simulation Parameters

selected probability and the remaining energy of these nodes (30). This protocol is adopted for its simplicity and because it is considered in many MWSN schemes $(1 ; 13)$. The number of $\mathrm{CH}$ is $5 \%$ of the total number sensor nodes. Nodes in regular observation generate periodic traffic using Constant Bit Rate (CBR) traffic model. The packet rate of these nodes is randomly set between 1 and 20 packets/sec to reflect low and moderate traffic; whereas nodes in emergency state generate bursty traffic using Poisson traffic model to produce random packets. The corresponding packet rate is set to 100 packets/sec to induce very high traffic.

The four protocols are assessed using OPNET simulator. The simulation parameters are summarized in Table 1.

\subsection{Simulation Results}

The compared schemes are evaluated with respect to their ability to adapt to CBSN traffic variations, as well as their ability to support mobility.

\subsubsection{Performance Under Traffic Variations}

The delay, packet drop and energy consumption of the four schemes are simulated to assess their ability to adapt to CBSN's traffic variations and to answer the corresponding traffic requirements. In this part, all nodes are considered to be static, and different traffic scenarios are assessed: when all nodes are in regular observation state, and when different number of nodes (5 to 30 nodes) enter into emergency state.

The average packet delay for the proposed TMAMAC scheme, ME-MAC, Mobisense, and TSEEC is presented in Figure 7. Simulation results show that when all nodes are in regular observation, generating periodic and relatively low traffic, TMA-MAC, Mobisense and TSEEC exhibit very close performances as they follow DTDMA slot allocation of different nodes. Delay in MEMAC is a slightly higher than the others because nodes have to contend to get channel access which induces more delay. Results also show that the proposed TMAMAC outperforms the other schemes when different number of nodes enter into emergency and thus generate bursty traffic. The reason is that TMA-MAC allows transmission of urgent data simultaneously over the same time slots at the beginning of the BP (over the ES period) using different spreading codes; this will guarantee immediate delivery of critical data without further delay even when the number of nodes carrying emergency data increases; and at the same time, it will decrease the number of slots allocated in the SS period, which will reduce the transmission delay of the periodic data generated by nodes in regular observation state, and will result in low average package delay. The delay induced by the proposed TMA-MAC slightly increases with the number of nodes entering into emergency as it needs to assign spreading codes to more nodes. As for ME-MAC, nodes transmit their data in different time slots based on traffic demand, and therefore, as the number of nodes carrying emergency data increases, the frame time expands, which will increase the delay of both emergency and regular traffic. Also, in ME-MAC, nodes send their request for data transmission over a contention-based period, which will lead to higher delays as nodes needs to listen to the medium to make sure that it is free prior to send their requests. The delay in ME-MAC significantly increases as the number of nodes entering emergency state reaches becomes high (above 15 nodes in this case), as the superframe saturates and leaves no more room to additional time slots; requests for data transmissions will be therefore declined, and force nodes to wait for a long time before finding available slots, which will intensely increase the packet delays. The delay performance of Mobisense is slightly better than ME-MAC as nodes do not compete to send slot allocation requests, however this delay is still high and becomes unmanageable when the number of nodes exceed a certain threshold due to superframe saturation. TSEEC outperforms ME-MAC and Mobisense due to better slot allocation process based on their priorities, and since it allows slots sharing by assigning unused slots when nodes do not have data to send to neighboring nodes. However, TSEEC under performs TMA-MAC even when the number of nodes with emergency traffic is low, since in TSEEC, the number of assigned slots for each node is proportional to the corresponding weighted value that depends on the location, battery, and priority of the nodes; since nodes with emergency data have higher priority than the others to transmit their data, their will have higher weighted values, and thus will be allocated more slots; the frame duration therefore increases with the number of nodes holding emergency traffic, which in return increases the packet delays. The superframe in TSEEC also saturates when the number of emergency nodes becomes high which significantly increments the delay.

The packet drop percentage simulation results are presented in Figure 8. They show that the proposed scheme outperforms the others and leads to lower packet drop percentage, specially when the number of nodes entering emergency becomes high. The reason is that in TMA-MAC, all nodes in emergency state are served 


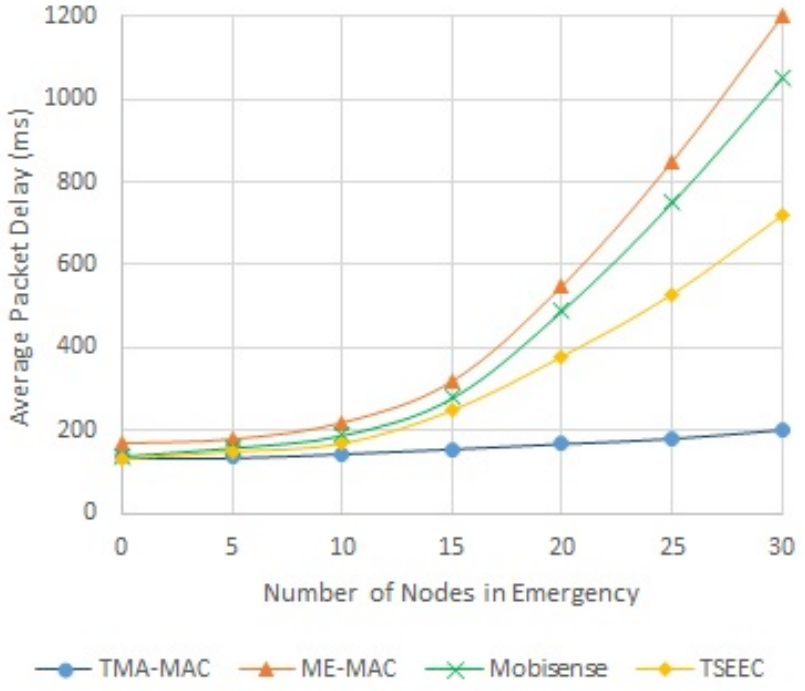

Figure 7: Delay Performance Under Various Number of Emergency Nodes

simultaneously at the beginning of the superframe, which allows the remaining nodes carrying periodic traffic to find available slots in the SS period; The probability of successful data transmission of both periodic an emergency data is therefore high due to high channel availability. ME-MAC induces the highest packet drop percentage due to the limited number of time slots leading to reduced channel availability, and due to the high contention for channel access when the traffic in the network increases. Mobisense also suffers from low channel availability when the number of nodes with emergency traffic increases, but performs better than ME-MAC since nodes do not compete for channel access. As for TSEEC, it outperforms both ME-MAC and Mobisense since it allows slots sharing which increases the channel availability. However, the superframe of TSEEC will eventually saturate when the number of nodes carrying emergency data becomes high, which will significantly increase the corresponding packet drop percentage.

The percentage of energy consumed by the compared MAC protocols with respect to the total energy consumption in the network is presented in Figure 9. Simulation results show that when all nodes are in regular observation state, TMA-MAC, mobisense, and TSEEC protocols perform similarly, as in the three schemes, nodes transmit their data each in a specific TDMA slots only, and remains asleep all the other times. ME-MAC consumes more energy than the others since nodes send their request for slot allocation over a contention-based period, which will lead to higher energy consumption due to the continuous listening to the medium to avoid collision. The energy consumption of TMA-MAC increases as the number of nodes carrying emergency data increase, due to the computational requirements needed to encode more packets generated by many nodes. However, the main traffic requirement

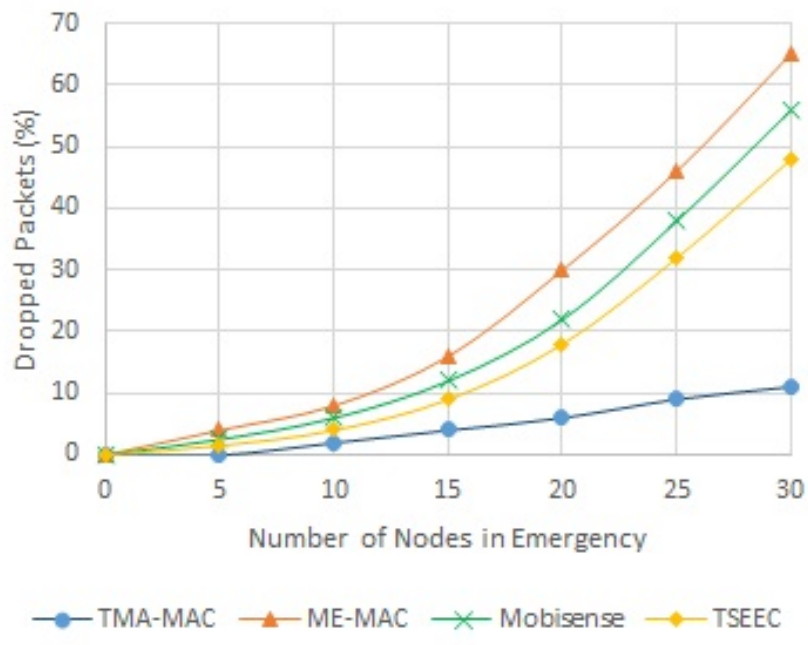

Figure 8: Packet Drop Performance Under Various Number of Emergency Nodes

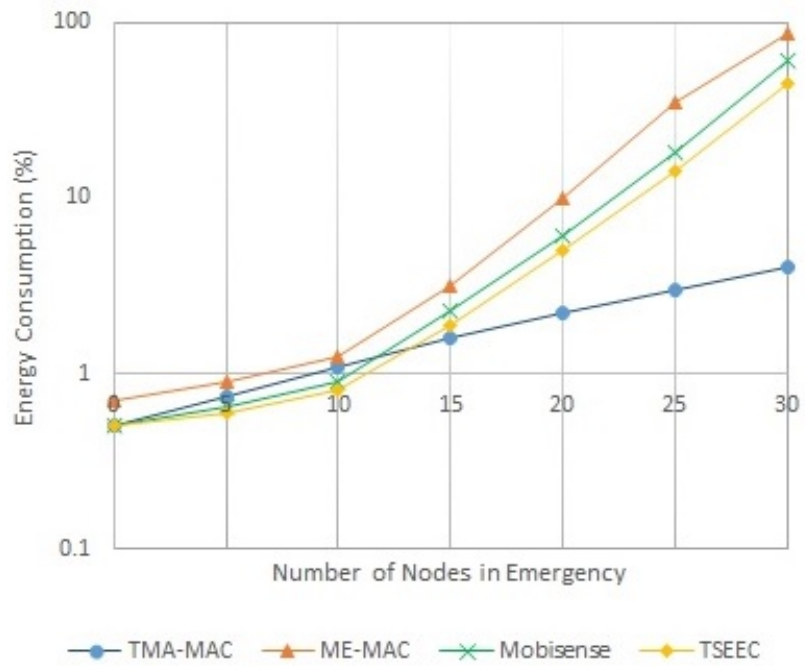

Figure 9: Energy Performance Under Various Number of Emergency Nodes

in CBSN is to deliver critical traffic instantly and reliably; therefore, the energy consumption of TMAMAC can be traded with its low delay and packet drop percentage feature. Also, in TMA-MAC, only the packets generated by nodes in emergency state will be assigned spreading codes; the periodic data is still transmitted over the SS period using TDMA mechanism, which will balance the corresponding energy consumption. As for ME-MAC, Mobisense, and TSEEC, their energy consumption increases with the number of nodes in emergency state, as more slots should be assigned in the frame causing them to stay awake for longer period of time. This energy consumption significantly increases when the protocols' superframe saturates (the number of emergency nodes becomes high), as nodes will need to remain active until finding available slots.

Simulation results showed that TMA-MAC represents an efficient and reliable scheme able to adapt 
to CBSNs traffic variations and address CBSNs traffic requirements, as it induces the lowest delay and packet drop percentage among the compared protocols, while trying to balance the energy consumption.

\subsubsection{Performance Under Mobility Variations}

To assess the performance of the four compared protocols in dynamic networks, we vary the level of mobility in the network by increasing the number of mobile nodes (from 5 to 35 nodes) and repeat simulations with respect to packet delay, packet drop percentage and energy consumption. In all scenarios, we consider that $30 \%$ of the nodes are in emergency state and thus generating bursty traffic.

The packet delay simulation results are illustrated in Figure 10. TMA-MAC outperforms the others and induces the lowest delay when the number of mobile nodes increases in the network. The reason is that TMAMAC gives priority to intra-cluster mobile nodes and allocates them slots in the MS period located before the SS period; also, in the MS period, it gives priority to mobile nodes with lowest RSSI to serve them the fastest possible way before the link breaks. This will decrease the delay of the mobile nodes, thus reducing the average packet delay in the network; in addition, TMA-MAC provides a mechanism to serve inter-cluster nodes in emergency state instantly, by allowing them to send join requests over the distributed EJR minislots throughout the BP; this will further reduce the delay of inter-cluster urgent packets and will lead to low network delay; this is in addition to adopting a common control channel that allows inter-cluster nodes to select the appropriate cluster to join quickly, and reduces the probability of failed join attempts which leads to decreased delay in the network. Results also show that the delay of TMA-MAC increases with the number of mobile nodes; this is due to the increased rate of join requests from inter-cluster mobile nodes with periodic traffic; such requests are addressed in the next frame which slightly increases the delay. The packet delay of ME-MAC is the highest among the compared schemes due to many factors: first, ME-MAC does not give priority to mobile nodes in slot assignment; therefore, the link with intra-cluster mobile nodes can break as they are not served fast enough; this will lead to inefficient use of slots and would therefore increase the packet delay. Second, increasing the network mobility level leads to more inter-cluster join requests; ME-MAC does not provide a mechanism to address the join requests of inter-cluster nodes carrying critical data quickly, and on the other hand, the inefficient slot allocation in MEMAC leads to quick superframe saturation, and would therefore increase the rate of join request rejections; these factors will further increment the delay. Third, the inter-cluster node in ME-MAC needs to listen to multiple channels to select the best cluster to join, which induces higher delays. And fourth, the increased number of mobile nodes will increase the contention in the

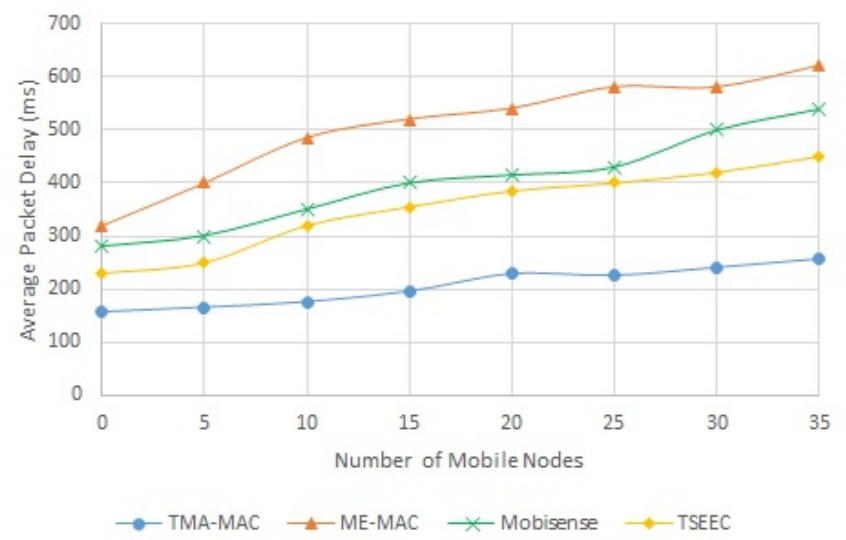

Figure 10: Delay Performance Under Various Number of Mobile Nodes

contention-based period, as this period is used to send both slot allocation requests, as well as join requests. The increased contention will also lead to more packet delays. Mobisense outperforms ME-MAC; In fact, like ME-MAC, Mobisense does not give priority to mobile nodes, does not have a mechanism to quickly handle urgent join requests; but unlike ME-MAC, Mobisense uses the common control channel mechanism for intercluster-nodes to efficiently find a new cluster, and intercluster nodes use allocated mini-slots at the end of the superframe to send their join request instead of contending between each other. As for TSEEC, it adopts a better slot allocation mechanism that allows sharing unused slots (due to link breakage for example) by other nodes, which will decrease the packets delay and leads to better performance than ME-MAC and Mobisense; however, TSEEC underperforms TMA-MAC since intercluster nodes' requests are addressed in the next frame even when nodes carry critical data, and also nodes should scan many channels to find the suitable cluster to join; which will lead to increased packet delays.

The simulation of the dropped packets percentage and the energy consumption percentage are presented in Figure 11 and Figure 12 respectively. Results

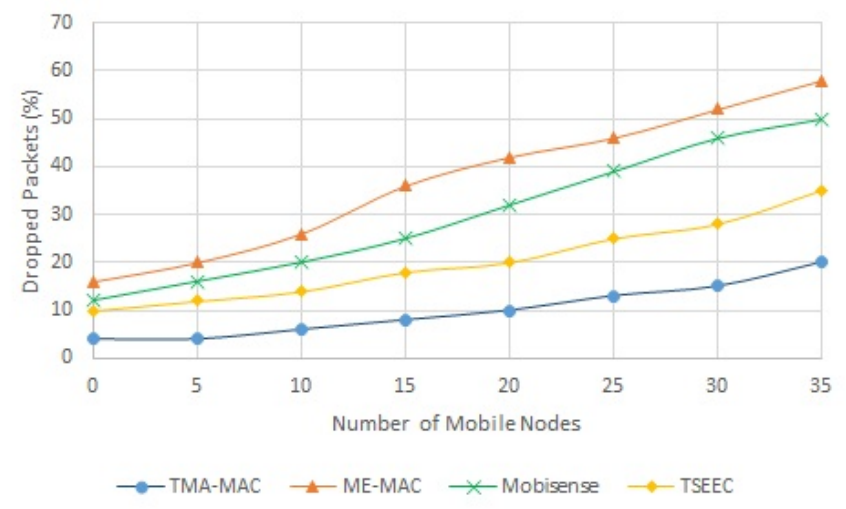

Figure 11: Packet Drop Performance Under Various Number of Mobile Nodes 


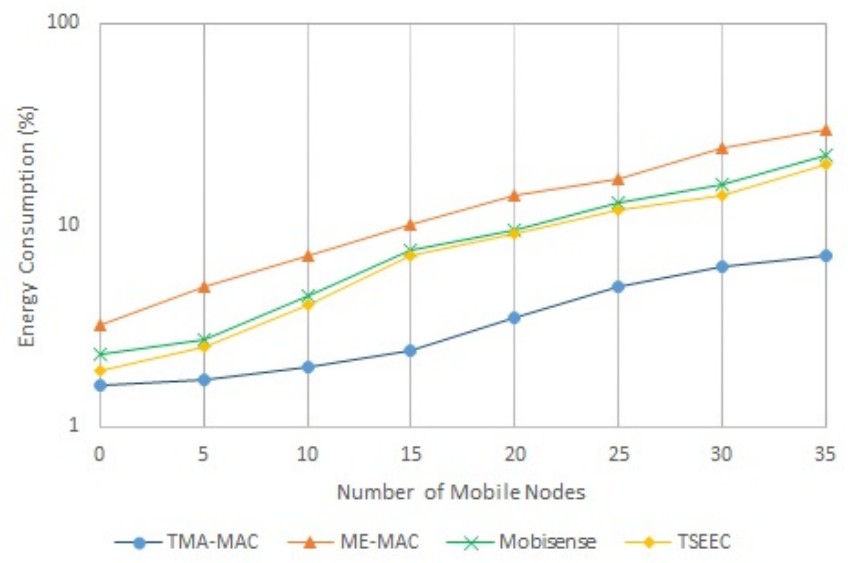

Figure 12: Energy Performance Under Various Number of Mobile Nodes

show that the proposed scheme is more reliable and consumes less energy among the compared schemes. The increased packet drop rate with the increased number of mobile nodes in ME-MAC and Mobisense is caused by the higher probability of link failure due to the slow service provided to intra-cluster mobile nodes; nodes will therefore re-transmit the packets which would raise the corresponding energy consumption; also, as more mobile nodes join the notwork, more join requests to clusters will be received; the inefficient slot allocation in ME-MAC and Mobisense leads to higher probability to reject join requests due to superframe saturation, which would increase the packet drop rate and consequently the energy consumed by these nodes to find appropriate cluster. However, Mobisense performs better than ME-MAC in terms of packet drop percentage and energy consumption, since inter-cluster nodes use a common channel to choose the appropriate cluster, which decreases the probability of failed attempts; these nodes also use assigned mini-slots at the end of the superframe without collision, which will reduce the packet drop rate and saves energy at the same time. TSEEC protocol induces less packet drop percentage than both ME-MAC and Mobisence, since it uses an efficient congestion control slot allocation that provides quick service of mobile nodes by assigning them priorities, and allows slots sharing; this will reduce the probability of link failure and leads to lower packet drop percentages and energy consumption; however, TSEEC do not use a common control channel for inter-cluster nodes to choose appropriate clusters, this will increase the energy consumed by listening to multiple channels prior of selecting the appropriate cluster to join; for this, the energy consumption performance of TSEEC is close to that of Mobisense. TMA-MAC outperforms the compared scheme as it adopts efficient slot allocation for intra-cluster mobile nodes to serve them as fast as possible before the link breaks; it also offers intercluster nodes with a common control channel to save energy and reduce probability of failed cluster join attempts; in addition, it provides a mechanism to serve inter-cluster nodes with critical data promptly, which will decrease their packet drop and re-transmission rates; and it provides high channel availability which reduces the probability of join requests failures, and decreases therefore the drop packets percentage and energy consumption.

The above results show that the proposed scheme offers a flexible and efficient mobility aware mechanism that can adapt to CBSN's dynamic traffic.

\section{Conclusion}

In this paper, an efficient hybrid Traffic and Mobility Aware MAC (TMA-MAC) protocol was proposed for CBSN. TMA-MAC is a traffic adaptive MAC that is able to address CBSN various traffic requirements by ensuring reliable delivery of critical traffic with the lowest delay, while maintaining low energy consumption during regular observation. TMA-MAC also provides a mechanism to efficiently handle CBSN nodes mobility by giving priority to intra-cluster mobile nodes over static nodes to send their data as fast as possible before the transmission link fails, and by adopting a common control channel that can be used by inter-cluster nodes to select the appropriate cluster to join, as well as allowing inter-cluster nodes holding critical data to send their join request over mini-slots distributed throughout the $\mathrm{BP}$, and granting them immediate channel access to transmit their data. The performance of TMA-MAC under both traffic and mobility variations was compared to other existing schemes. Simulations of packet delay, percentage of packet drops and energy consumption were conducted, when the number of nodes entering emergency state is varied between 0 and 30 nodes, and when the number of mobile nodes is increased from 0 to 35 nodes. Results showed that TMA-MAC outperformed the compared protocols as it induced the lowest delay, packet drop percentage and energy consumption. It presents therefore an efficient and reliable protocol that can address CBSNs traffic and mobility requirements. Future work includes proposing an efficient routing protocol for CBSN and applying the TMA-MAC on that protocol to propose an optimal MAC/routing platform for CBSN. Also, further simulations can be performed to study the performance of the proposed TMA-MAC with respect to other metrics like scalability and throughput, in addition to addressing other CBSN challenges like channel fading.

\section{Acknowledgment}

This work was supported by the EIPHI Graduate School(contract ANR-17-EURE-0002), the Hubert Curien CEDRE programme (n40283YK), and by the Lebanese University Research Program (Number: 4/6132). 


\section{References}

[1] Yahya, B. and Ben-Othman, J.: 'An adaptive mobility aware and energy efficient MAC protocol for wireless sensor networks', IEEE Symposium on Computers and Communications, 2009. ISCC 2009, 2009, pp. 15-21, IEEE.

[2] Kacso, A. and Abbas Kazmi, W.: 'MAC Protocol Design Requirements for Mobilityaware Wireless Sensor Networks', The Eleventh International Conference on Sensor Technologies and Applications. Sensorcomm, 2017.

[3] Gondha, A.V. and Bavarva, A.A.: 'A Review on Mobility and Mobility Aware MAC Protocols in Wireless Sensor Network', International Journal of Computer Applications, vol. 91, number 2, 2014, Foundation of Computer Science.

[4] Boudargham, N. and Bou abdo, J. and Demerjian, J. and Guyeux, C. and Makhoul, A.: 'Collaborative Body Sensor Networks: Taxonomy and Open Challenges', Middle East \& North Africa COMMunications Conference (MENACOMM), 2018, IEEE.

[5] Boudargham, N. and Bou abdo, J. and Demerjian, J. and Guyeux, C. and Makhoul, A.: 'Efficient Hybrid Emergency Aware MAC Protocol for Wireless Body Sensor Networks',Sensors Journal, vol. 18, number 10, pp. 3572, 2018, Multidisciplinary Digital Publishing Institute.

[6] Salayma, M. and Al-Dubai, A. and Romdhani, I. and Nasser, Y.: 'Reliability and Energy Efficiency Enhancement for Emergency-Aware Wireless Body Area Networks (WBAN)',IEEE Transactions on Green Communications and Networking, 2018, IEEE.

[7] Liu, B. and Yan, Z. and Chen, C.W.: 'MAC protocol in wireless body area networks for E-health: Challenges and a context-aware design', IEEE Wireless Communications journal, vol. 20, number 4, pp. 64-72, 2013, IEEE.

[8] Salayma, M. and Al-Dubai, A. and Romdhani, I.: 'Reliability and energy efficiency enhancement in wireless body area networks for e-health', 19th Conference of Open Innovations Association (FRUCT), 2016.

[9] Deepshikha and Chauhan, S.: 'Succinct Review on MAC Protocols for Wireless Body Area Networks',International Journal of Grid and Distributed Computing, vol. 11, number 1, pp. 65-79, 2018, SCIENCE \& ENGINEERING RESEARCH SUPPORT SOC RM 402, MAN-JE BLDG, 449-8 OJUNG-DONG, DAEDOEK-GU, DAEJON, 00000, SOUTH KOREA.
[10] Zareei, M. and Islam, A.K.M. and VargasRosales, C. and Mansoor, N. and Goudarzi, S. and Rehmani, M. H.: 'Mobility-aware Medium Access Control Protocols for Wireless Sensor Networks: A Survey',Journal of Network and Computer Applications, vol. 104, 2017, doi $=$ 10.1016/j.jnca.2017.12.009.

[11] Silva, R. and Silva, J.S and Boavida, F.: 'Mobility in wireless sensor networks-survey and proposal', Computer Communications journal, vol. 52, pp. 1-20, 2014, Elsevier.

[12] Boudargham, N. and Bou abdo, J. and Demerjian, J. and Guyeux, C. and Makhoul, A.: 'Investigating Low Level Protocols for Wireless Body Sensor Networks',13th ACS/IEEE International Conference on Computer Systems and Applications, 2016, IEEE.

[13] Ali, M. and Suleman, T. and Uzmi, Z. A. and others: 'MMAC: a mobility-adaptive, collision-free MAC protocol for wireless sensor networks',IPCCC, vol. 28, number 2, pp. 401-407, 2005.

[14] Raja, A. and Su, X.: 'A mobility adaptive hybrid protocol for wireless sensor networks', Consumer Communications and Networking Conference, 2008. CCNC 2008. 5th IEEE, pp. 692-696, 2008, IEEE.

[15] Gonga, A. and Landsiedel, O. and Johansson, M.: 'MobiSense: Power-efficient micro-mobility in wireless sensor networks',International Conference on distributed Computing in Sensor Systems and Workshops, pp. 1-8, 20011, IEEE.

[16] hmad, A. and Jabbar, S. and Paul, A. and Rho, S.: 'Mobility aware energy efficient congestion control in mobile wireless sensor network', International Journal of Distributed Sensor Networks, vol. 10, number 3, pp. 530416, 2014, SAGE Publications Sage UK: London, England.

[17] Pham, H. and Jha, S.: 'An adaptive mobilityaware MAC protocol for sensor networks (MSMAC)',International Conference on Mobile Ad-hoc and Sensor Systems, pp. 558-560, 2004, IEEE

[18] Zareei, M. and Taghizadeh, A. and Budiarto, R. and Wan, T.: 'EMS-MAC: energy efficient contentionbased medium access control protocol for mobile sensor networks', The Computer Journal, vol. 54, number 12, pp. 1963-1972, 2011, Oxford University Press.

[19] Choi, S. and Lee, J. and Kim, Y.: 'An adaptive mobility-supporting MAC protocol for mobile sensor networks', Vehicular Technology Conference, 2008. VTC Spring 2008, pp. 168-172, 2018, IEEE.

[20] Hameed, S.A. and Shaaban, E.M. and Faheem, H.M. and Ghoniemy, M.S.: 'Mobility-aware 
MAC protocol for delay-sensitive wireless sensor networks',International Conference on Ultra Modern Telecommunications \& Workshops, 2009. ICUMT'09, pp. 1-8, 2009, IEEE.

[21] Nadeem, Q. and Javaid, N. and Mohammad, S.N. and Khan, M.Y. and Sarfraz, S. and Gull, M.: 'Simple: Stable increased-throughput multi-hop protocol for link efficiency in wireless body area networks',8th International Conference on Broadband and Wireless Computing, Communication and Applications (BWCCA), 2013.

[22] Tauqir, A. and Javaid, N. and Akram, S. and Rao, A. and Mohammad, S.N.: 'Distance aware relaying energy-efficient: Dare to monitor patients in multihop body area sensor networks',8th International Conference on Broadband and Wireless Computing, Communication and Applications (BWCCA), pp. 206-213, 2013. 2013.

[23] Khan, A. and Memon, A. A. and Memon, A.A.: 'Time Synchronization for Mobile Wireless Sensor Network',International Journal of Computer and Electrical Engineering, vol. 6, number 2, 2014, IACSIT Press.

[24] Alam, M.M. and Hamida, E. B. and Berder, O. and Menard, D. and Sentieys, O.: 'A heuristic self-adaptive medium access control for resourceconstrained WBAN Systems',IEEE Access, vol. 4, npp. 1287-1300, 2016, IEEE.

[25] 'Single Chip $2.4 \mathrm{GHz}$ Transceiver',Nordic Semiconductor, number nRF24L01, July 2007, Rev. 2.0 .

[26] Boudargham, N. and Bou abdo, J. and Demerjian, J. and Guyeux, C.: 'Exhaustive Study on Medical Sensors', The 11th International Conference on Sensor Technologies and Applications (SENSORCOM), September 2017, Rome, Italy.

[27] Boudargham, N. and Bou abdo, J. and Demerjian, J.and Guyeux, C. and Atechian, T.: 'Efficient Cluster-Based Routing Algorithm for Body Sensor Networks',Middle East \&6 North Africa COMMunications Conference (MENACOMM), 2018, IEEE.

[28] Jhumka, A. and Kulkarni, S.: 'On the design of mobility-tolerant TDMA-based media access control (MAC) protocol for mobile sensor networks',International Conference on Distributed Computing and Internet Technology, pp. 42-53, 2007, Springer.

[29] Elson, Jeremy and Girod, Lewis and Estrin, Deborah: 'Fine-grained network time synchronization using reference broadcasts', $A C M$ SIGOPS Operating Systems Review, vol. 36 pp. 147-163, 2002, ACM.
[30] Heinzelman, W.B. and Chandrakasan, A. and Balakrishnan, H.: 'An application-specific protocol architecture for wireless microsensor networks',IEEE Transactions on wireless communications, vol. 1, number 4, pp. 660-670, 2002,IEEE. 